\title{
Work function control of transparent ITO/Ag/ITO multilayer using nano-sized Ag and Al top layer
}

\author{
Hyo-Joong Kim, Ki-Won Seo and Han-Ki Kim* \\ Department of Advanced Materials Engineering for Information and Electronics, Kyung Hee University, \\ 1 Seocheon-dong, Yongin, Gyeoggi-do 446-701, Republic of Korea \\ Corresponding author: e-mail: imdlhkkim@khu.ac.kr
}

\begin{abstract}
We report on the effect of nano-sized $\mathrm{Ag}$ and $\mathrm{Al}$ layer on the electrical and optical properties of the ITO/Ag/ITO (IAI) multilayer films as well as a work function. With increasing the thickness of the Ag and Al layer, we investigated the change of electrical and optical properties of the IAI multilayer in detail. Regardless of thickness of the $\mathrm{Ag}$ and $\mathrm{Al}$ top layer, the IAI multilayer showed a constant sheet resistance of $\sim 5 \mathrm{Ohm} / \mathrm{square}$ and a high transmittance of $\sim 87 \%$. However, the surface morphology and microstructure of the IAI multilayer were affected by the nano-sized $\mathrm{Al}$ and $\mathrm{Ag}$ cover layer due to different oxidation behavior. The work function of the IAI multilayer with nano-sized Ag cover layer is constant $(\sim 4.95 \mathrm{eV})$ regardless of $\mathrm{Ag}$ thickness, while the work function of the IAI multilayer with Al layer decreased from $4.95 \mathrm{eV}$ to $4.75 \mathrm{eV}$ with increasing $\mathrm{Al}$ thickness. This indicates that the coating of the nano-sized $\mathrm{Al}$ is effective way to decrease the work function of the IAI multilayer films.
\end{abstract}

Key words: Nano-sized Ag and Al layer, ITO/Ag/ITO, Oxidation, Work function

\section{Introduction}

As a promising transparent electrode with a low resistivity and high optical transparency, a transparent oxide/metal/oxide (OMO) multilayer structure has been widely investigated in organic light emitting diodes (OLEDs) and organic solar cells (OSCs) because they provide better optical and electrical characteristics than those of attainable with a single-layer ITO film [1-5]. Until now, nano-sized Ag interlayer-based OMO schemes, such as ITO/Ag/ITO (IAI), IZO/Ag/IZO, IZTO/Ag/IZTO, $\mathrm{GZO} / \mathrm{Ag} / \mathrm{GZO}, \mathrm{AZO} / \mathrm{Ag} / \mathrm{AZO}$ and $\mathrm{ZTO} / \mathrm{Ag} / \mathrm{ZTO}$ films, have been suggested due to their low resistivity comparable to those of metal films and high optical transmittance caused by the antireflection effect [6]. Although IAI multilayer can be prepared with a low resistivity, a high transparency, and a large are on a glass or flexible substrate using a batch-type sputtering or rollto-roll sputtering, their integration into OLEDs and OPVs with a well-defined electronic energy level is critically affected by work function of the IAI multilayer. Therefore, engineering of the work function of the IAI multilayer is important to apply the IAI in high-efficient OLEDs and OPVs [7-9]. In general, the work function of the IAI multilayer significantly increased by plasma treatment or ozone treatment because the work function of top ITO layer in the IAI is easily affected by plasma or ozone treatment $[10,11]$. However, detailed investigation to decrease the work function of the IAI multilayer is still lack. Therefore, decreasing of the work function of IAI multilayer is one of the most important issues to apply the IAI multilayer as transparent cathode in OLEDs and OPVs.

In this work, we suggested a simple way to decrease the work function of IAI multilayer using a nano-sized Al layer. The approach involves the evaporation of $\mathrm{Ag}$ and $\mathrm{Al}$ layer on the IAI multilayer, followed by measurement of the work function of the IAI multilayer using by ultra violet photoelectron spectroscopy (UPS). In addition, we investigated the effect of nano-sized $\mathrm{Ag}$ and $\mathrm{Al}$ layer on the electrical, optical, and morphological and structure properties of the IAI multilayer as a function of $\mathrm{Ag}$ and $\mathrm{Al}$ thickness.

\section{Experiment}

The ITO/Ag/ITO (IAI) multilayer films were prepared on glass substrate using a direct current (DC) magnetron sputtering with multi cathode guns at room temperature. Using tilted cathode guns, the bottom ITO, $\mathrm{Ag}$, and top ITO layers were continuously deposited on glass substrate without breaking the vacuum. A commercial ITO target (10 wt.\% $\mathrm{SnO}_{2}$-doped $\mathrm{In}_{2} \mathrm{O}_{3}$ ) was used to sputter both bottom and top ITO layers. An optimized $35 \mathrm{~nm}$-thick bottom ITO layer was sputtered at a constant DC power of $100 \mathrm{~W}$, an $\mathrm{Ar} / \mathrm{O}_{2}$ flow ratio of $20 / 0.3 \mathrm{sccm}$, and a working pressure of 2 mTorr onto a $15 \times 15 \mathrm{~mm}^{2}$ glass substrate. After sputtering the bottom ITO layer, thin Ag interlayer was continuously sputtered on the bottom ITO layer using commercial Ag target at a constant DC power of $100 \mathrm{~W}$. Finally, the top ITO layer was sputtered onto the Ag layer under same sputtering conditions to those used for the bottom ITO layer. After coating of IAI multilayer, Ag or Al layer was evaported on the IAI multilayer as a function of thickness $(0.3$ and $0.5 \mathrm{~nm})$ respectively. The electrical properties of the $\mathrm{Ag}$ or $\mathrm{Al}$ covered IAI multilayer were characterized using Hall measurements as a function of $\mathrm{Ag}$ or Al thickness. Using a UV/visible spectrometer, the optical transmittances of the $\mathrm{Ag}$ or $\mathrm{Al}$ covered IAI multilayer were measured at wavelengths between 300- 
$800 \mathrm{~nm}$. The surface morphology and structure of the $\mathrm{Ag}$ or Al covered IAI mutilayer was examined using a field emission scanning electron microscope (FESEM)and Xray diffraction (XRD) as a function of $\mathrm{Ag}$ or $\mathrm{Al}$ thickness. UPS measurement was performed to investigate the change of work funtion of the IAI multilyer as a function of the thickenss of nano-sized Ag or Al layer. The spectra were measured with a ESCALAB 210 system UV (He I) discharged sources, and the based pressure of the analysis chamber was $2 \times 10^{-10}$ Torr. The spectra were measured with a sample bias of $-10 \mathrm{~V}$ (cutoff region) to accelerate the low energy secondary electrons.

\section{Results and Discussion}

Figure 1(a) and (b) show electrical properties of the IAI multilayer with $\mathrm{Ag}$ and $\mathrm{Al}$ layer as a function of thickness. In spite of evaporation of $\mathrm{Ag}$ and $\mathrm{Al}$ layer, the IAI multilayer showed similar sheet resistance and resistivity to those of as-deposited IAI multilayer. This indicates that evaporation of nano-sized Ag or Al layer cannot affect on the electrical properties of the IAI multilayer because the main conduction path of IAI multilayer is metal $\mathrm{Ag}$ interlayer between bottom and top ITO layers [12]. However, carrier mobility of the IAI multilayer with nanosized Ag or Al layer slightly decreased due to scattering between $\mathrm{Ag}$ or Al layer and top ITO layer. The carrier concentration of the IAI multilayer slightly increased with evaporation of $\mathrm{Ag}$ or $\mathrm{Al}$ layer because the nano-size $\mathrm{Ag}$ or Al layer can provide electrons into the top ITO layer.

Figure 1 (c) and (d) show the optical transmittance of IAI multilayer with evaporated $\mathrm{Ag}$ or $\mathrm{Al}$ layer as a function ofl thickness. The identical optical transmittance $(\sim 87.9 \%)$ of the IAI multillayer with $\mathrm{Ag}$ or Al layer at a wavelength of $550 \mathrm{~nm}$ to that $(\sim 86.9 \%)$ of the bare-IAI multilayer indicates that the existence of nano-sized Ag or Al layer on the top ITO layer cannot affect the optical transmttance of the IAI multilayer.

Figure 2 (a) and (b) compared the surface morphology of IAI multilayer before and after evaporation of the $\mathrm{Ag}$ or $\mathrm{Al}$ layer. In case of the IAI multilayer with 0.3 and $0.5 \mathrm{~nm}$ thick Ag layer, they showed different surface morphology with that of bare-IAI multilayer as shown inset in Fig 2(a) due to agglomeration of $\mathrm{Ag}$ layer. However, it is noteworthy that the surface morphology of the IAI multilayer with 0.3 and $0.5 \mathrm{~nm}$ thick Al layer showed identical surface to that of bare-IAI multilayer. The similar surface mophology of the IAI multilayer with nano-sized Al layer to bare IAI multilayer could be attributted to the easy oxidation of Al layer on the top ITO layer [13]. Figure 2(c) and (d) show the XRD plots of IAI multilayer before and after $\mathrm{Ag}$ or $\mathrm{Al}$ evaporation. In case of bare IAI multilayer, it shows ITO (222), ITO (400), and $\mathrm{Ag}$ (111) peaks at $2 \theta=30,33$ and $38^{\circ}$. The XRD plots of IAI multilayers with 0.3 and $0.5 \mathrm{~nm}$ thick Ag layer also show ITO (222) and Ag (111) peaks. Due to the overlap of $\mathrm{Ag}$ (111) peaks, it is difficult to distinguish the $\mathrm{Ag}$ interlayer peak and nano-sized $\mathrm{Ag}$ top layer peak. (a)

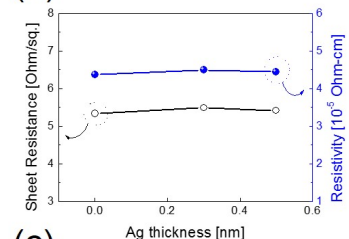

(c)

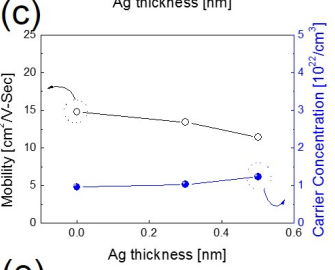

$(\mathrm{e})$

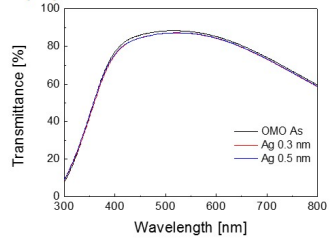

(b)

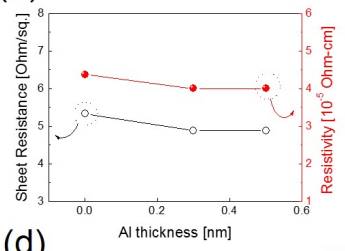

(d)

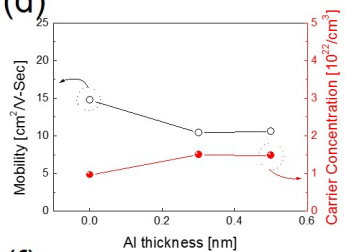

(f)

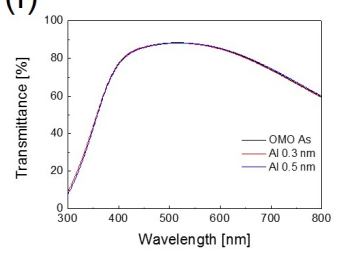

Figure 1. Sheet resistance, resistivity, carrier mobility, and concentration of the IAI multilayer with evaporated (a) Ag and (b) Al layer as a function of thickness. Optical transmittance of the IAI multilayer with 0.3 and $0.5 \mathrm{~nm}$ thick (c) Ag and (d) $\mathrm{Al}$ evaporated layer.

However, there is no additional peak except crystalline ITO and Ag peak indicating no interfacial reaction between nano-sized Ag top layer and ITO layer. However, the IAI multilayer with 0.3 and $0.5 \mathrm{~nm}$ thick Al layer showed different XRD plot with the bare-IAI multilayer. Compared to XRD plot of bare IAI multilayer, it shows additional $\mathrm{Al}_{2} \mathrm{O}_{3}(104)$ peak at $2 \theta=35^{\circ}$ due to oxidation of evaprated $\mathrm{Al}$ layer on the top ITO layer.

Figure 3 shows the UPS spectra in the secondary electron cutoff region as a function of $\mathrm{Ag}$ or $\mathrm{Al}$ thickness on the IAI multilayer. As shown in Figure 3(a), similar the secondary electron cutoff indicate that evaporation of 0.3 and $0.5 \mathrm{~nm}$ thick Ag layer cannot change the work function of the IAI multilayer. However, the secondary electron cutoff of the IAI multilayer with Al layer shifts from $16.25 \mathrm{eV}$ to $16.45 \mathrm{eV}$ with increasing $\mathrm{Al}$ thickness. Compared to work function $(4.95 \mathrm{eV})$ of the bare-IAI multilayer, the work function of IAI multilayer with 0.3 and $0.5 \mathrm{~nm}$ thick $\mathrm{Al}$ layer is $4.85 \mathrm{eV}$ and $4.75 \mathrm{eV}$, respectively. The change of work function in IAI multilayer could be attributed to the oxidation of Al layer as expected from XRD results. In general, $\mathrm{AlO}_{\mathrm{x}}$ exhibits a lower work function than clean Al layer [14]. Therefore, the oxidation of Al layer on ITO layer plays a important role in reducing the work function of the IAI multilayer. 
(a)

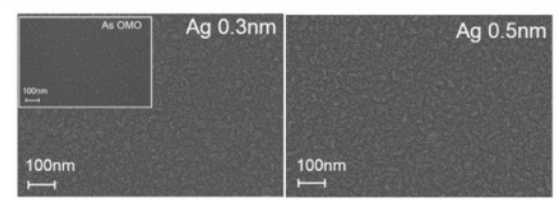

(b)

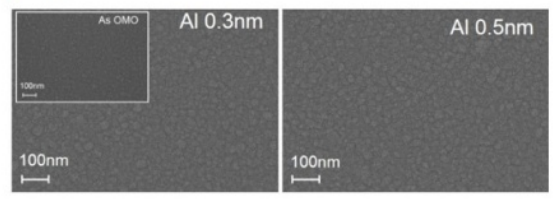

(c)

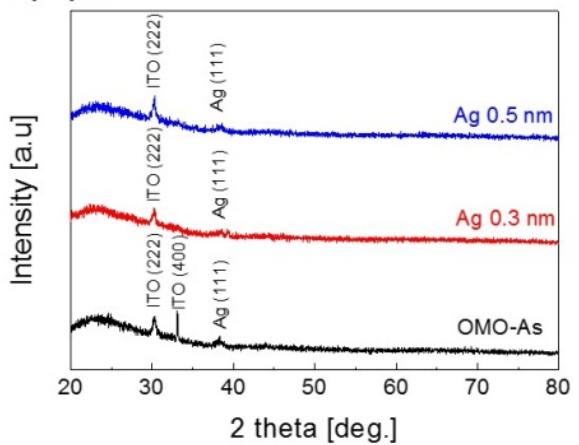

(d)

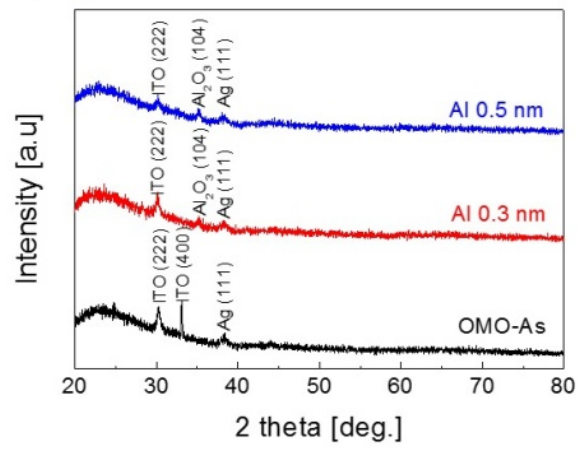

Figure 2. Surface FESEM images of IAI multilayer with 0.3 and $0.5 \mathrm{~nm}$ thick (a) Ag and (b) Al layer with inset showing surface morphology of the bare-IAI multilayer. Comparison of XRD plots of the IAI multilayer with 0.3 and $0.5 \mathrm{~nm}$ thick (c) $\mathrm{Ag}$ and (d) Al layer. (a)
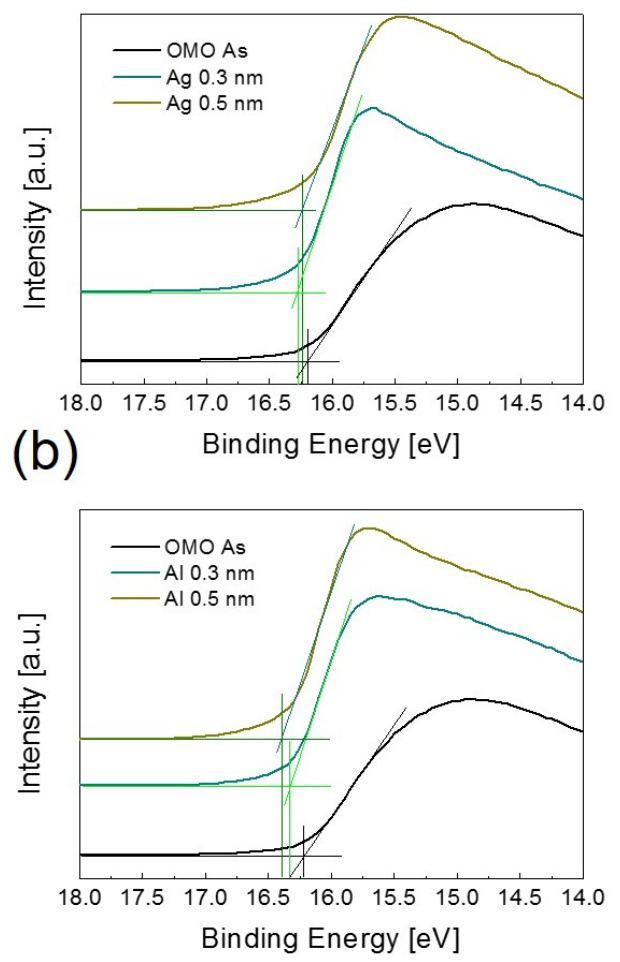

Figure 3. The UPS spectra of the IAI multilayer with nano-sized (a) Ag and (b) Al layer as a function of Ag or Al thickness.

\section{Conclusions}

We developed a simple method for controlling the work function of transparent IAI multilayer using nano-sized $\mathrm{Ag}$ and $\mathrm{Al}$ layer. Regardless of thickness of the Ag and Al top layer, the IAI multilayer showed a constant sheet resistance of $\sim 5 \mathrm{Ohm} / \mathrm{square}$ and a high transmittance of $\sim 87 \%$. However, the surface morphology and microstructure of the IAI multilayer were affected by the nano-sized $\mathrm{Al}$ and $\mathrm{Ag}$ layer due to different oxidation behavior. The work function of the IAI multilayer with nano-sized Ag cover layer is constant $(\sim 4.9 \mathrm{eV})$ regardless of Ag thickness, while the work function of the IAI multilayer with Al layer decreased from $4.95 \mathrm{eV}$ to 4.75 $\mathrm{eV}$ with increasing $\mathrm{Al}$ thickness. This indicates that the coating of the nano-sized $\mathrm{Al}$ is effective way to decrease the work function of the IAI multilayer films.

\section{Acknowledgments}

This work was supported by the Industrial Strategic technology development program (10042412).

\section{Reference}

[1] J-A. Jeong, J.-Y. Lee, and H.-K. Kim, Electrochem. Solid State Lett., 12, J105-J108 (2009)

[2] J.-W. Lim, S. J. Kang, S. Lee, J. J. Kim, and H.-K. Kim, J. Appl. Phys., 112, 023513 (2012)

[3] S. Y. Ryu et al., Appl. Phys. Lett., 92, 023306 (2008)

[4] K.-H. Choi, H.-W. Koo, T.-W. Kim, and H.-K. Kim, Appl. Phys. Lett., 100, 263505 (2012)

[5] Y.-S. Park, K.-H. Choi, and H.-K. Kim, J. Phys. D : 
Appl. Phys., 42, 235109 (2009)

[6] A. Kloppel, W. Kriegseis, B. K. Meyer, A. Scharmann, C. Daube, J. Stollenwerk, and J. Trube, Thin Solid Films, 365, 139-146 (2012)

[7] C. Y. Jiang, X. W. Sun, D. W. Zhao, A. K. K. Kyaw and Y. N. Li, Sol. Energy Mater. Sol. Cells, 94, 1618-1621 (2013)

[8] M. G. Helander, Z. B. Wang, J. Qiu, M. T. Greiner, D. P. Puzzo, Z. W. Liu and Z. H. Lu, Science, 332, 944 (2011) [9] H.-K. Kim, and S. J. Kang, J. Korean Phys. Soc., 47, S417-S421 (2005)

[10] C. Kim, B. Lee, H. J. Yang, H. M. Lee, J. G. Lee, and H. Shin, J. Korean Phys. Soc., 47, S417-S421 (2005)

[11] B.-S. Kim, D.-E. Kim, Y.-K. Jang, N.-S. Lee, O.-K. Kwon, and Y.-S. Kwon, J. Korean Phys. Soc., 50, 18581861 (2007)

[12] H.-J. Kim, K.-W. Seo, Y.-J. Noh, S.-I. Na, and H.-K. Kim, J. Vac. Sci. Technol. A, 32, 051507 (2005)

[13] T. R. B. Fong, A. Sellinger, and X. Hu, ACS Nano, 2, 2250-2256 (2008)

[14] K. L. Wang, B. Lai, M. Lu, X. Zhou, L. S. Liao, X. M. Ding, S. T. Lee, and X. Y. Hou, Thin Solid Films, 363, 178-181 (2000)

(Received January 31, 2015; Accepted June 1, 2015) 